\section{Nutraceuticals: Separating the wheat from the chaff}

Andrea T. Borchers $\square$ Carl L. Keen a Judy S. Stern $\square$ M. Eric Gershwin

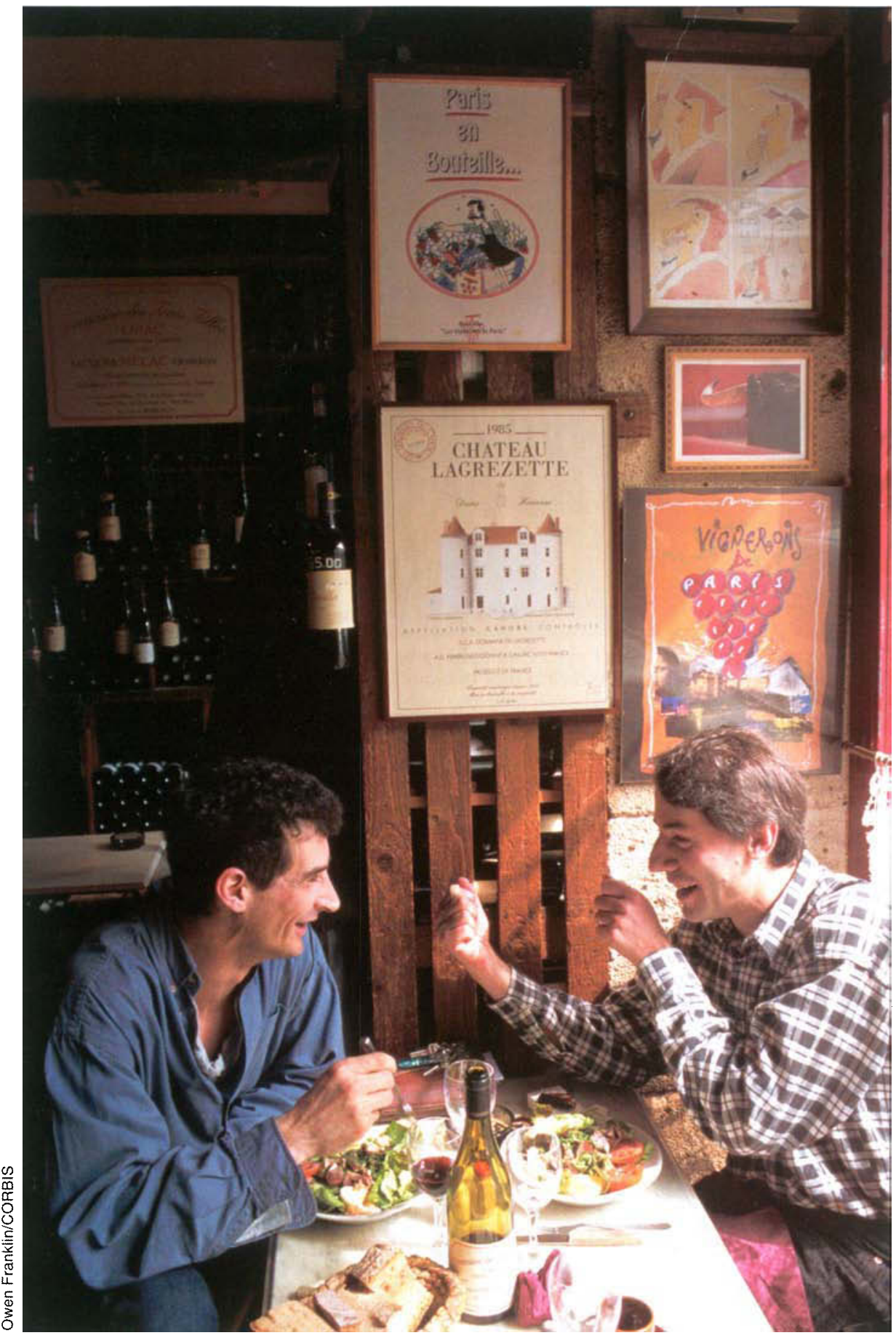

The "French paradox" continues to puzzle scientists. In France, consumption of saturated fats is high, but heart disease is lower than expected. The antioxidants in red wine may offer an explanation.
Foods provide nutrients such as vitamins, minerals, protein, carbohydrate and fat and a host of other nonessential nutrients that may confer health benefits. Some "nutraceuticals" have been found to boost the immune system, enhance memory function and possibly slow the aging process. For example, flavonoids - which are found in red wine, purple grape juice, green tea and cocoa products - exhibit potent antioxidant activity in laboratory experiments and have been postulated to protect against coronary artery disease and reduce the risk of cancer. Recognizing potential health benefits from flavonoids and other plant extracts, some manufacturers are creating "functional" foods by fortifying, bioengineering and otherwise modifying foods so that they contain higher than normal concentrations of these components. With the exception of echinacea, St. John's wort and Ginkgo biloba, there is a paucity of scientific data for the majority of botanicals sold in health food stores and supermarkets. At the same time, adverse reactions to some botanicals have been documented in humans. Many would argue that government regulation of botanicals is inadequate. Further studies and comprehensive databases are needed to establish the safety and efficacy of popular and widely consumed dietary supplements.

\footnotetext{
Tn recent years, evidence has accuImulated that foods provide not only nutrients (vitamins, minerals, protein, carbohydrate and fat), but also a host of non-nutrient components that may confer health benefits.

Recognizing this, some manufacturers have begun creating functional foods (see glossary, facing page) by fortifying, bioengineering or otherwise modifying foods so that they contain higher than normal concentrations of these components. Others are marketing the extracts of certain foods -
} 
such as garlic, parsley and cranberries — in the form of pills, capsules, tablets or liquids. In addition, certain individual phytochemicals are available in health food stores and even in supermarkets. One example is the near ubiquitous plant constituent, quercetin. Most commonly, food extracts are sold as dietary supplements. As such, they are in the same category as many vitamin and mineral supplements as well as many botanical (herbal) extracts. A dietary supplement was defined by the U.S. Congress as:

"A product (other than tobacco) that is intended to supplement the diet that contains one or more of the following dietary ingredients: a vitamin, a mineral, an herb or other botanical, an amino acid, a dietary substance for use by man to supplement the diet by increasing the total daily intake, or a concentrate, metabolite, constituent, extract or combinations of these ingredients" (FDA 1994).

\section{Markets for supplements expand}

During the past decade, the market for botanical supplements has exploded, fueled in part by claims that select botanicals can do everything from curing colds to improving memory and facilitating weight loss.

Market data reflect the rapid growth of consumer interest in dietary supplements. The dietary supplement market experienced unprecedented reported increases in dollar sales of approximately $50 \%$ between September 1997 and September 1998 (Kruger 1998). The majority of this increase was attributable to the sales of botanical supplements. Continuing shortterm growth rates of at least $20 \%$ per year have been predicted (Radice 1998; Scimone and Scimone 1998).

Diet, nutrition and lifestyle changes together constitute one of the seven categories of complementary and alternative medicine (CAM); herbal medicine is another (table 1). The increased popularity of dietary and herbal supplements parallels the growing interest in and use of CAM practices. Between 1990 and 1997, the percentage of people who had used some form of CAM within the previous 12 months rose from $34 \%$ to $42 \%$ (Eisenberg et al. 1993; Eisenberg et al. 1998).

In view of the frequent use of CAM therapies in general, and dietary supplements in particular (nutritional, nutraceutical and herbal), it is vital to establish the safety and efficacy of such approaches. For the majority of botanicals sold in health food stores and supermarkets, there is an astounding paucity of scientific data. Notable exceptions include echinacea, St. John's wort and Ginkgo biloba. For these three botanicals, clinical studies have demonstrated that they are, by and large, safe and can have some beneficial effects. However, not all safety aspects have been investigated. Some recent findings on St. John's wort, for example, indicate that caution is necessary when botanicals are taken together with prescription and over-the-counter drugs. In one case, St. John's Wort was associated with reduced concentrations of certain protease inhibitors used to treat AIDS; in the other, scientists reported St. John's wort decreased effectiveness of a drug used to reduce tissue rejection in heart transplant patients (Piscitelli et al. 2000; Ruschitzka et al 2000).

Echinacea reportedly helps to lower the incidence of upper respiratory tract infections and reduce their symptoms (Melchart et al. 1998). St. John's wort has been an effective treatment for mild to moderate clinical depression (Wheatley 1997; Laakmann et al. 1998; Lenoir et al. 1999) and Ginkgo biloba has slowed the deterioration of patients with dementias, particularly Alzheimer's disease, in numerous randomized, placebo-controlled, doubleblind clinical studies (Kleijnen and Knipschild 1992; Kanowski et al. 1996; Le Bars et al. 1997).

\section{Vitamin data inconclusive}

Considerable research in recent years has been devoted to determining which single chemical constituent (or group of structurally similar constituents) is responsible for a specific health-promoting effect of a plant extract or food. A large body of evidence supports the concept that increased
Numerous patients who are treated with prescription and other drugs simultaneously consume a variety of botanicals, often without informing their physicians.

\section{Glossary of terms}

Botanical supplement: Plantderived supplement.

Herbal supplement: The subset of botanical supplements derived from herbaceous plants.

Chemopreventive agent: Essential or nonessential nutrient or other agent shown to prevent cancers or metastases in animal models and/or humans.

Designer food: Foods processed to enhance their texture, taste or health benefits, or some combination of these characteristics (e.g., low-fat ice cream).

Dietary supplement: Food product ingested to correct a perceived deficit in the overall diet; typically not a whole food.

Functional food: Food that provides health benefits beyond energy and essential nutrients (e.g., yogurt, which promotes beneficial microflora in the gut).

Nutraceutical: Food or food product that decreases the risk of disease establishment or progression.

Nutrient: Anything that is ingested, digested and metabolized.

Essential nutrient: Nutrients that must be ingested for the survival of the species.

Nonessential nutrient: Nutrients not necessary for survival of the species that may have a health benefit nonetheless (e.g., catechins, which have potent antioxidant activity).

Phytochemical: Substances found in plants and plant-derived products. 
consumption of fruits and vegetables can be associated with decreased risk for a variety of cancers as well as coronary artery disease (Potter and Steinmetz 1996). As a result, the constituents of fruits and vegetables that are believed responsible for these health benefits are often called "nutraceuticals."

This definition also applies to essential nutrients and nonessential nutrients that modulate the immune system, enhance memory function and potentially slow the aging process. The antioxidant vitamins $\alpha$-tocopherol (vitamin E) and ascorbic acid (vitamin C) may be partially responsible for the beneficial effects of fruits and vegetables. Indeed, epidemiological studies indicate that the intake level of these vitamins correlates with a reduced risk of cancer and heart disease. However, a favorable effect of vitamin C supplements - as opposed to consumption of foods rich in vitamin $\mathrm{C}$ on cardiovascular morbidity and mortality has not been documented in randomized, prospective studies. Similarly, of four trials examining the effect of vitamin E supplements on these parameters, only one reported positive

TABLE 1. Seven categories of alternative medicine

Alternative systems of medical practice: Health care ranging from self-care according to folk principles, to care rendered in an organized health-care system based on alternative traditions of practice (e.g., acupuncture, food taboos).

Bioelectromagnetic applications: The study of how living organisms interact with electromagnetic fields.

Diet, nutrition, lifestyle changes: The knowledge of how to prevent illness, maintain health and reverse the effects of chronic disease through dietary or nutritional intervention.

Herbal medicine: Employing plant and plant products from folk medicine traditions for pharmacological use

Manual healing: Using touch and manipulation with the hands as a diagnostic and therapeutic tool (e.g., chiropractic medicine, acupressure).

Mind/body control: Exploring the mind's capacity to affect the body, based on traditional medical systems that make use of the interconnectedness of mind and body

Pharmacological/biological treatments: Drugs and vaccines not yet accepted by mainstream medicine.

Source: National Center for Complementary and Alternative Medicine, http://nccam.nih.gov/nccam fcp/classify. Accessed Sept. 2000. effects while three others obtained neutral results (Institute of Medicine 2000).

The recent report of the Standing Committee on the Scientific Evaluation of Dietary Reference Intakes, therefore, concluded that existing data do not adequately answer the question of whether increased intake of vitamin $C$ and/or vitamin $E$ from supplements reduces the risk of coronary artery disease (Institute of Medicine 2000).

\section{Red wine and the French paradox}

Similarly, the polyphenols (a broad class of compounds found in plants) have garnered increasing scientific interest in recent years. It is wellestablished that polyphenols, in particular flavonoids, are powerful antioxidants in numerous in vitro systems - those using test tubes and cell cultures rather than animal or human tests. In many of these systems, flavonoids such as quercetin, kaempferol and the catechins exhibit several-fold more potent antioxidant activity than $\alpha$-tocopherol (Terao et al. 1994; Lotito and Fraga 1998). Antioxidants reduce risk of oxidative damage to molecules, protecting cells and tissues. Flavonoids can be found in foods as diverse as wine, blueberries, onions and chocolate.

The "French paradox," an observation that was based on epidemiology, provided a renewed impetus to study polyphenols. In the early 1980 s, scientists reported that the incidence of coronary artery disease in France was lower than in comparable nations while, paradoxically, the intake of saturated fat was quite high. One explanation advanced for this paradox was the high consumption of red wine in France (Stanley and Mazier 1999). Drinking other forms of alcohol provided much less protection than regular intake of red wine. Therefore, it was suggested that the flavonoids in red wine, rather than the alcohol content, were one of the candidates explaining its cardioprotective effects.

Since then, a number of epidemiological studies have reported an association between higher flavonoid intake from fruits and vegetables - as well as products prepared from them, such as wine, grape juice and apple juice - and lowered risk of cardiovascular disease (Knekt et al. 1996; Hertog et al. 1993; Hertog et al. 1995). A weaker association has also been observed with decreased risk of certain cancers (Hertog et al. 1995).

To what extent are these compounds absorbed and what metabolic changes do they undergo? Over the last few years, research from a variety of laboratories has established that flavonoids are at least partially absorbed (Borchers et al. 2000). The extent of absorption depends not only on the structure of the individual flavonoid but also on the form (with or without attached carbohydrate groups) in which it is ingested. Once absorbed, flavonoids are almost immediately and extensively modified by the addition of methyl, sulfur and/or carbohydrate groups. In addition, some flavonoids, in particular quercetin, bind avidly to certain serum proteins, especially albumin. Few studies have investigated how, and to what extent, metabolic changes and serum-protein binding affect the bioavailability and bioactivity of flavonoids. It remains unclear how valid the findings are from in vitro studies, which are conducted using unmetabolized flavonoids without attached carbohydrates, and for in vivo situations, in which they are administered to living animals and human beings.

\section{Beneficial flavonoids in cocoa}

Evidence is slowly accumulating that dietary supplementation with flavonoids has cardioprotective effects in experimental animals. Similar findings have been reported after supplementation with flavonoid-rich substances such as red wine, purple grape juice, green tea and cocoa products. In humans, flavonoid-rich foods have been shown to reduce oxidative damage to lipids (Kondo et al. 1996) and platelet reactivity (Rein et al. 2000). Both processes have been implicated in the development and progression of cardiovascular disease. However, the observed effects cannot be clearly attributed to flavonoids since wine, tea and cocoa contain numerous other constituents including other antioxi- 
dants and immunomodulatory factors. One or more of these, singly or in combination, might be partly or wholly responsible for the beneficial effects.

These and other studies suggest that the amount of flavonoids ingested by eating the recommended portions of at least five servings of fruits and vegetables a day may not be sufficient to provide optimal cardioprotective or chemopreventive effects. This is particularly true if the fruits and vegetables are low in the flavonoids that provide these health effects. An important goal for research in the next decade is to identify which foods provide flavonoids and in what quantities. That raises the question of whether it is advisable at this point in time to fortify, bioengineer or otherwise modify foods, creating functional foods to increase the content of flavonoids or other health-promoting substances.

\section{Chemical complexity of plants}

Many of the animal studies have been done with isolated flavonoids. This does not reflect the way flavonoids and other phytochemicals are normally consumed, either as part of whole plant-based foods or botanical extracts from whole plants. This approach ignores a variety of issues that arise from the chemical complexity of plants.

One indication of this complexity is that isolated plant ingredients - even those thought to be $a$, or even the bioactive constituent - often have different effects than the whole-plant extract, suggesting that interactions occur among various chemical constituents of a particular extract. For example, certain chemical components of a plant food or extract might

enhance but could also inhibit the absorption of one or more other constituents.

Individual ingredients or groups of ingredients may also promote or interfere with the metabolism of other chemicals. Constituents that might themselves be inert may still serve important functions by stabilizing or otherwise interacting with bioactive components.

Through all of these mechanisms, plant constituents have the potential to increase or decrease not only the bioavailability but also the potential toxicity of other ingredients. Of course, these activities are not restricted to other components of the same plant, but extend to other plants and foods, prescription and over-thecounter drugs, and even environmental toxins. These observations take on particular importance because numerous patients, treated with prescription and other drugs, simultaneously consume a variety of botanicals, often without informing their physicians (Eisenberg et al. 1998).

\section{Dearth of botanicals research}

Little systematic research has been conducted concerning the interactions among plant constituents, or between plant constituents and prescription or over-the-counter drugs.

Some case reports highlight that such interactions can occur and have harmful or even fatal consequences, for example, with interactions between St. John's wort and AIDS drugs. Researchers observed that the concentration of the HIV-I protease inhibitor indinavir was reduced $50 \%$ in individuals who took St. John's wort. (Piscitelli et al. 2000). In two heart transplant patients, treatment with St. John's wort was associated with a decrease in plasma cyclosporin concentrations, leading to symptoms of acute transplant rejection (Ruschitzka et al. 2000). After discontinuing the St. John's wort, plasma concentrations of cyclosporin returned to a therapeutic level with no further symptoms of transplant rejection.

Investigations of the activities of plant foods or extracts, including the 


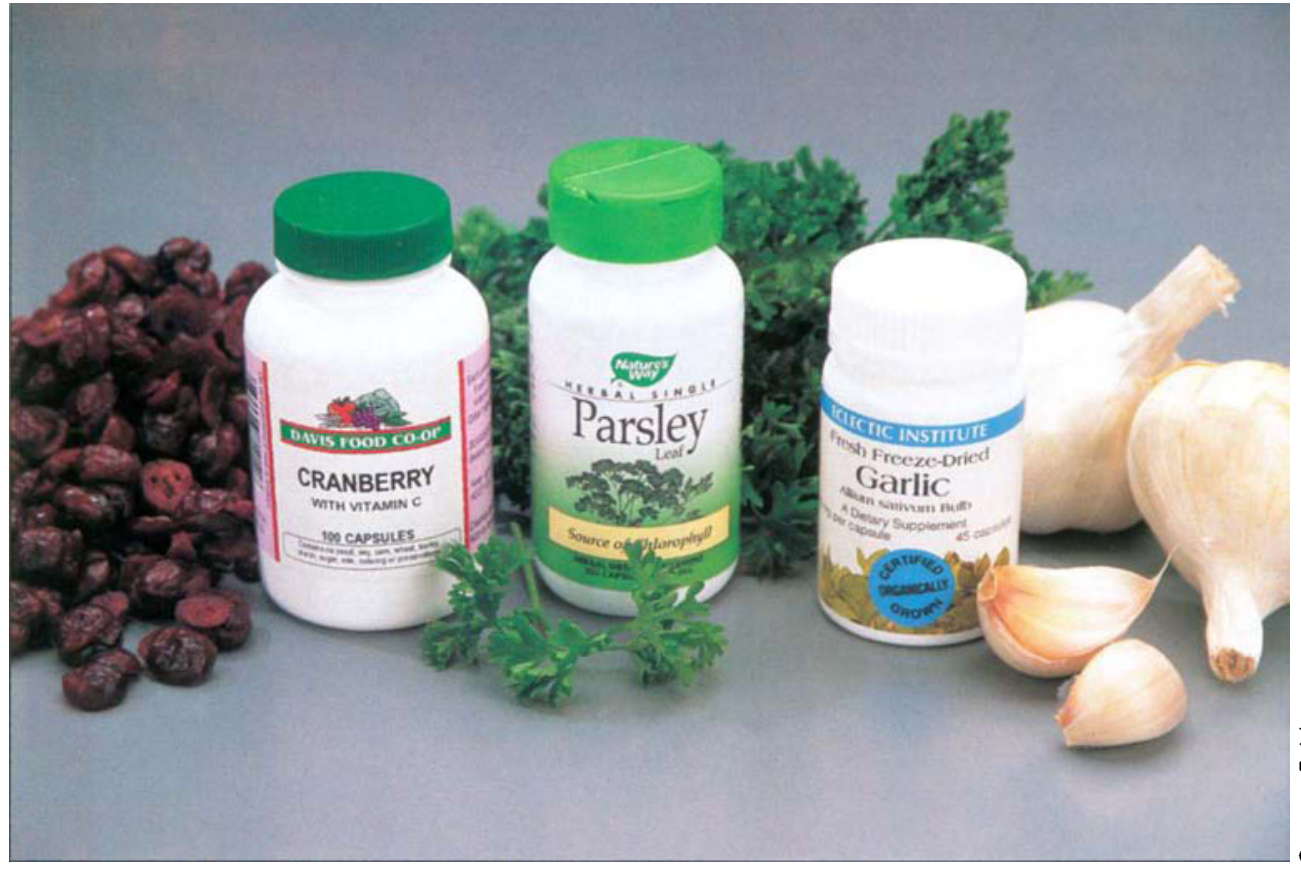

"Real" fruits and vegetables such as cranberries, parsley and garlic are now widely available in pill form - as botanical extracts from plants. However, little systematic research has been conducted on the interactions between such supplements and over-the-counter and prescription drugs.

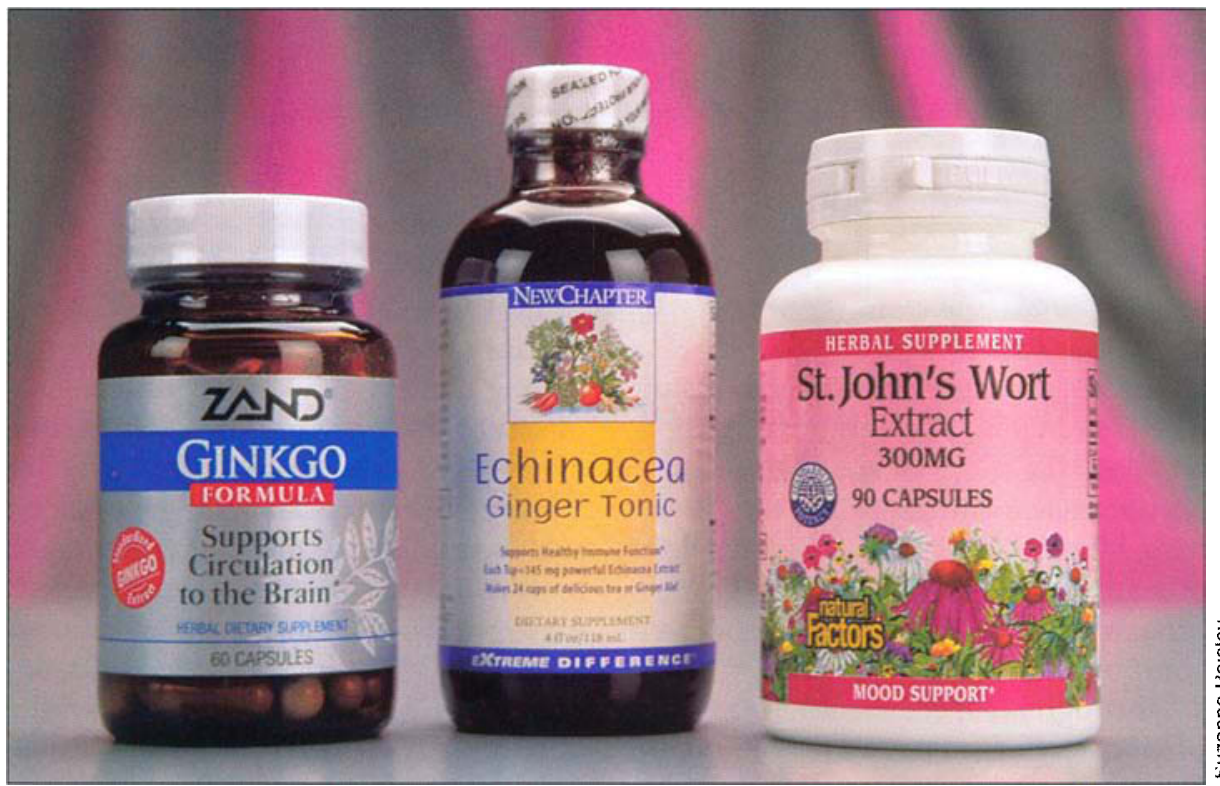

Gingko biloba, echinacea and St. John's wort are among the few dietary supplements that have undergone clinical studies. For the most part, these three have been found safe and effective, but some interactions with other drugs have been reported.

above-mentioned interactions, have been impeded by several factors.

First, we are far from having a complete list of all the biologically active components of a single plant, much less a list of all its chemical constituents. In addition, the exact chemical composition of a plant can vary considerably with the method of growing, harvesting, processing and storage. Minor differences in the overall

\section{Standardization lacking}

It has also been shown that the composition of different brands of the same herbal product can vary by factors of 2 or more, with some containing little or none of the known active compounds (Consumer Reports 1995).

The dietary supplement market has experienced astonishing increases in sales in recent years. There are indications, however, that the growth rate for this market segment is slowing from the $20 \%$ predicted in 1998 to about $8 \%$ (Wilhelm 1999). The credibility of the industry may have suffered from media reports $\frac{\vec{y}}{\frac{a}{a}}$ that the potency of several widely sold botanicals was not what the product label claimed. The industry as a whole may also have lost some credibility because of poor quality control by individual manufacturers.

Botanical supplements are marketed as dietary supplements and, as such, are regulated by the U.S. Food and Drug Administration with criteria that are less strict than those for prescription and over-the-counter drugs. We agree with other analysts that existing regulation is not only inadequate, but also inadequately enforced (Wilhelm 1999; Williamson and Wyandt 1999). In the interest of consumers, it is crucial to ensure that all manufacturers follow good manufacturing practices (GMPs), FDA standards enforced in some segments of the food industry. Enforcing GMPs could ensure that botanical preparations contain what is listed on the label, at the concentrations stated, and are not contaminated with other substances.

In 1994, Congress passed the Dietary Supplement Health and Education Act (DSHEA), which amended the Federal Food, Drug and Cosmetic Act to specifically include provisions for dietary supplements and their ingredients. Dietary supplements, like other foods, must bear ingredient labeling. As with other food products, it is the responsibility of the manufacturer to ensure that its products are safe and properly labeled prior to marketing. The Food and Drug Administration (FDA) is responsible for overseeing not only the safety, but also the label- 
ing, package inserts and accompanying literature of dietary supplements.

DSHEA provides specific instructions as to what a product label must and must not contain. In the case of dietary supplements, the label must identify the product as a "dietary supplement." The label must further include information regarding the name and quantity of each ingredient. The label of a product that contains botanical ingredients must provide information as to the part of the plant from which the ingredient is derived. If a product does not contain what the label proclaims it does, the product is fraudulent. Importantly, a supplement cannot lay claim to "diagnose, cure, mitigate, treat or prevent disease," as those are the intended uses of FDAapproved drugs. Since dietary supplements do not have to undergo FDA approval, such claims would make the supplement an unauthorized, that is, illegal, drug. However, DSHEA specifically allows retailers to provide "third-party" materials to help inform consumers about any health-related benefits of dietary supplements.

Unfortunately, much of the existing research on foods or plant extracts suffers from the failure of researchers to provide any chemical analysis of products they used. When conflicting results are obtained by different researchers, it is difficult, if not impossible, to determine whether discrepancies are due to differences in the products or to other experimental factors unrelated to the product.

The great variability in the content of active ingredients in herbal extracts has prompted numerous scientists, public-health advocates and agencies to call for their standardization. This means that for each lot of a botanical, the extraction procedure is adjusted to ensure that a predetermined amount of a known active ingredient, or group of ingredients, is present in every capsule, pill, tablet or bottle of liquid extract. Good as this concept may sound, there are nevertheless a variety of problems associated with it. Most of these concerns are directly related to the issue of interactions among various constituents of a plant extract.
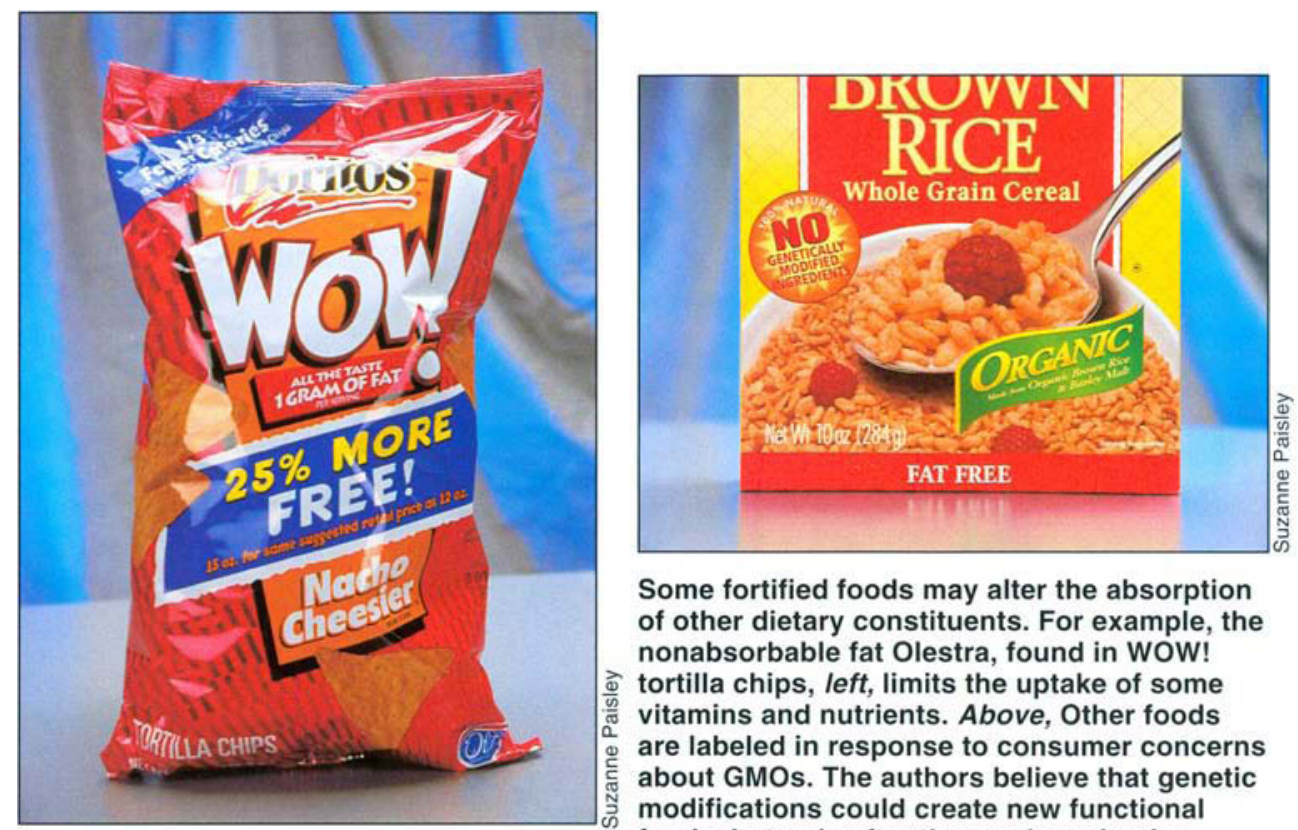

Some fortified foods may alter the absorption of other dietary constituents. For example, the nonabsorbable fat Olestra, found in WOW! tortilla chips, left, limits the uptake of some vitamins and nutrients. Above, Other foods are labeled in response to consumer concerns about GMOs. The authors believe that genetic modifications could create new functional foods, but only after thorough evaluation.

\section{Safe upper limits unknown}

The creation of certain functional foods can be viewed as equivalent to the standardization of an herbal extract. This would be the case if, through bioengineering, fortification or other modifications, manufacturers ensured that a specified amount of one particular chemical or group of chemicals, such as a polyphenol or a group of polyphenols, is present in the final product.

Just like standardization of herbal extracts, this approach ignores the vast potential for as yet largely unknown and uninvestigated chemical and biochemical interactions. It further ignores that we have very little information on "safe upper limits" for phytochemicals, the maximum amounts that can be consumed without any deleterious effects. The potential for harmful effects, for example, has been shown with the polyphenol quercetin. At high doses, this flavonoid can promote the growth of certain types of cancer in experimental animals, although it has been shown to prevent other kinds of cancers (Borchers et al. 2000).

Is it advisable to fortify, bioengineer or otherwise modify or create functional foods in order to increase their content of flavonoids or other substances thought to promote health? We would argue that the time may be ripe to develop such products. How- ever, there must be a clear appreciation that such products would need to be carefully evaluated for safety and efficacy before introducing them into the marketplace.

\section{Allergies to modified foods}

Another safety issue that needs to be addressed is whether fortified and modified foods have altered allergenicity, as has been reputed with soy protein. In addition, fortified foods may alter absorption of other dietary constituents. For example, some have suggested that the intake of high amounts of the nonabsorbable fat Olestra may be cause for concern. Olestra product labels must carry the government-mandated warning, "Olestra may cause abdominal cramping and loose stools." The discovery that Olestra also inhibits the absorption of some vitamins and other nutrients, while not proven to be a problem, illustrates the type of issue that requires further research.

\section{Future in focus: Comprehensive databases are needed}

The current reductionist approach to science may be inadequate to cope with the inordinate complexity of plant foods and extracts and their activities in living systems. We envision the creation during the next two decades of large computer databases with the majority of data available on 


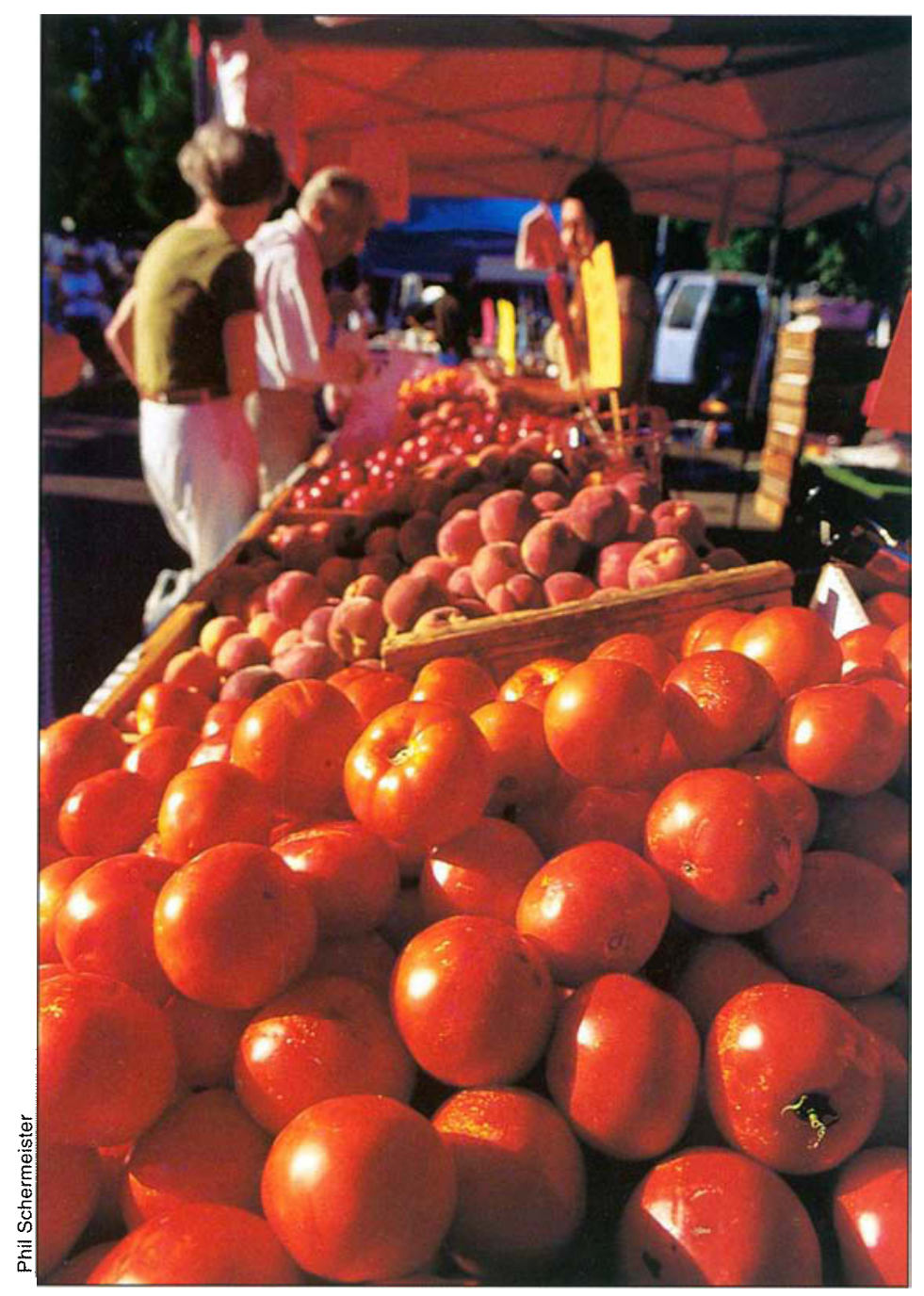

a particular plant food or extract. Once such databases exist, we envision the application of computer analyses and the modeling of chemical and biochemical interactions using the intellectual frameworks of fuzzy logic and control theories.

Ultimately, a critical challenge to educators and public-health officials will be the development of educational programs that can transmit this information to the lay public in a digestible fashion. The above can only be accomplished through the efforts of interdisciplinary teams of researchers and educators.

A.T. Borchers is Postdoctoral Fellow, C.L. Keen is Chair and Professor, and J.S. Stern is Professor, Department of Nutrition, UC Davis; and M.E. Gershwin is Chief and Professor, Division of Rheumatology, Allergy and Clinical Immunology, UC Davis.

The exact chemical
composition of
plants - and the ex-
tracts derived from
them - can vary
considerably. Conse-
quently, scientists,
public-health advo-
cates and regulators
have called for stan-
dardization of
supplements, so that
predetermined
amounts of active
botanical ingredients
are present.

\section{References}

Borchers AT, Keen $\mathrm{CL}$, Hannum S, Gershwin ME. 2000 (in press). Cocoa and chocolate: Composition, bioavailability and health implications. $\mathrm{J}$ Med Food.

Consumer Reports 1995. Herbal roulette 60:11. p 698-705.

Eisenberg DM Davis RB, Ettner SL, et al. 1998. Trends in alternative medicine use in the United States, 1990-1997: Results of a follow-up national survey. JAMA 280:1569-75.

Eisenberg DM, Kessler RC, Foster C, et al. 1993. Unconventional medicine in the

United States: Prevalence, costs and patterns of use. N Engl J Med 328:246-52.

[FDA] U.S. Food and Drug Administration. 1994. Dietary Supplement Health and Education Act of 1994. http://vm.cfsan.fda.gov/ $\sim$ dms/dietsupp.html. Accessed October 2000.

Hertog MGL, Feskens EJM, Hollman $\mathrm{PCH}$, et al. 1993. Dietary antioxidant flavonoids and risk of coronary heart disease: The Zutphen elderly study. Lancet

\section{2:1007-11.}

Hertog MGL, Kromhout D, Aravanis C, et al. 1995. Flavonoid intake and long-term risk of coronary disease and cancer in the seven countries study. Arch Intern Med 155:381-6.

Institute of Medicine, Standing Committee on the Scientific Evaluation of Dietary Reference Intakes, Food and Nutrition Board.

2000. Dietary Reference intakes for Vitamin C, Vitamin E, Selenium and Carotenoids. National Academy Press, Washington DC.

Kanowski S, Herrmann WM, Stephan K, et al. 1996. Proof of efficacy of the Ginkgo biloba special extract EGb 761 in outpatients suffering from mild to moderate primary degenerative dementia of the Alzheimer type or multi-infarct dementia. Pharmacopsychiatry 29:47-56.

Kleijnen J, Knipschild P. 1992. Ginkgo biloba for cerebral insufficiency. $\mathrm{Br} \mathrm{J}$ Clin Pharmacol 34:352-8.
Knekt $P$, Jarvinen $R$, Reunanen $A$ Maatela J. 1996. Flavonoid intake and coronary mortality in Finland: A cohort study. Brit Med J 312:478-81.

Kondo K, Hirano R, Matsumoto A, et al. 1996. Inhibition of LDL oxidation by cocoa. Lancet 348:1514.

Kruger RM. 1998. The herb garden grows. Discount Merchandiser 1998:45-7.

Laakmann G, Schule C, Baghai T, Kieser M. 1998. St. John's wort in mild to moderate depression: The relevance of hyperforin for the clinical efficacy. Pharmacopsychiatry 31(Suppl 1):54-9.

Le Bars PL, Katz MM, Berman N, et al. for the North American EGb Study Group. 1997. A placebo-controlled, double-blind, randomized trial of an extract of Ginkgo biloba for dementia. JAMA 278:1327-32.

Lenoir S, Degenring FH, Saller R. 1999. A double-blind randomised trial to investigate three different concentrations of a standardised fresh plant extract obtained from the shoot tips of Hypericum perforatum L. Phytomedicine 6:141-6.

Lotito SB, Fraga CG. 1998. (+)-Catechin prevents human plasma oxidation. Free Rad Biol Med 24:435-41.

Melchart D, Walther $E$, Linde $K$, et al. 1998. Echinacea root extracts for the prevention of upper respiratory tract infections. Arch Fam Med 7:541-5.

Mizuno M, Yamada J, Terai H, et al. 1994. Differences in the immunomodulating effects between wild and cultured Panax ginseng. Biochem Biophys Res Commun 200:1672-8.

Piscitelli SC, Burstein AH, Chaitt D, et al. 2000. Indinavir concentrations and St. John's wort. Lancet 355:547-8.

Potter JD, Steinmetz K. 1996. Vegetables, fruit and phytoestrogens as preventive agents. In: Stewart BW, McGregor D,

Kleihues $\mathrm{P}$ (eds.). Principles of Chemoprevention. Lyon, France: IARC Scientific Publications. p 61-90.

Radice C. 1998. Herbal poll. Progressive Grocer (May): 141.

Rein D, Paglieroni TG, Wun T, et al. 2000 Cocoa inhibits platelet activation and function. Am J Clin Nutr 72:30-5.

Ruschitzka R, Meier PJ, Turina M, et al. 2000. Acute heart transplant rejection due to St. John's wort. Lancet 355:548-9.

Scimone A, Scimone A. 1998. U.S. sees the green in herbal supplements. Chemical Market Reporter (Jul 13):FR3-4.

Stanley LL, Mazier MJP. 1999. Potential explanations for the French paradox. Nutr Res 19:3-15.

Terao J, Piskula M, Yao Q. 1994. Protective effect of epicatechin, epicatechin gallate and quercetin on lipid peroxidation in phospholipid bilayers. Arch Biochem Biophys 308:278-84.

Wheatley D. 1997. LI 160, an extract of St. John's wort, versus amitriptyline in mildly to moderately depressed outpatients: A controlled 6-week clinical trial. Pharmacopsychiatry 30(Suppl 2):77-80

Wilhelm C. 1999. Growing pains in botanicals and herbal supplements. Chemical Market Reporter (Nov 8):FR12-3.

Williamson JS, Wyandt CM. 1999. The herbal generation: Legal and regulatory considerations. Drug Topics (Apr 19):101-10. 